\title{
EFECTIVIDAD DE UN PROGRAMA EDUCATIVO SOBRE EL CONOCIMIENTO DE SEXUALIDAD EN ADOLESCENTES. MOCHE, 2015
}

\author{
EFFECTIVENESS OF AN EDUCATIONAL PROGRAM IN THE \\ KNOWLEDGE ON SEXUALITY IN ADOLESCENTS. \\ MOCHE, 2015
}

Janet J. Chunga ${ }^{1}$, Nora Vargas ${ }^{2}$, V. Soledad García ${ }^{3}$ Flor Márquez

\section{RESUMEN}

La presente investigación tuvo como objetivo establecer la relación que existe entre un programa educativo y el nivel de conocimiento sobre sexualidad en adolescentes de instituciones educativas del distrito de Moche. La investigación de diseño cuantitativa, pre experimental, correlacional, de corte transversal, estuvo conformada por una muestra de 96 adolescentes, a quienes se les aplicó dos instrumentos para la recolección de datos: El cuestionario para determinar el nivel de conocimiento sobre sexualidad y el programa educativo "Reconociendo mi adolescencia. Los datos fueron procesados con el software estadístico SPSS v22.0. Para establecer la relación entre las variables de estudio se aplicó la prueba estadística " $t$ " de student

1 Maestra en Salud Pública, mención Planificación y Gestión en la Universidad Nacional de Trujillo. Docente Asociada del Departamento Académico de Enfermería de la Mujer y el Niño de la Facultad de Enfermería. Universidad Nacional de Trujillo.

2 Maestra en Enfermería, mención Salud de la Mujer y el Niño en la Universidad Nacional de Trujillo. Docente Asociada del Departamento Académico de Enfermería de la Mujer y el Niño de la Facultad de Enfermería. Universidad Nacional de Trujillo.

3 Maestra en Enfermería Mención Salud de La Mujer y el Niño en la Universidad Nacional de Trujillo. Docente Asociada del Departamento Académico de Enfermería de la Mujer y el Niño de la Facultad de Enfermería. Universidad Nacional de Trujillo.

4 Maestra en Salud Pública, mención Planificación y Gestión en la Universidad Nacional de Trujillo. Docente Asociada del Departamento Académico de Enfermería de la Mujer y el Niño de la Facultad de Enfermería. Universidad Nacional de Trujillo. 
considerando un nivel de significancia de 0,05. Se llegó a las siguientes conclusiones respecto al nivel de conocimiento sobre sexualidad en adolescentes antes de la aplicación del programa educativo el 78,1\% alcanzó un nivel medio de conocimientos, el 17,7\% un nivel bajo y el 4,2\% un nivel alto, después de la aplicación del programa educativo el 59,4\% alcanzó un nivel medio de conocimientos, $38,5 \%$ un nivel alto y el 2,1\% un nivel bajo. En relación a las variables de estudio se demostró que existe relación estadística altamente significativa entre ellas.

PALABRAS CLAVE: Adolescente; conocimiento; sexualidad (DeCS BIREME).

\section{ABSTRACT}

The research is quantitative, pre-experimental, correlational, cross-sectional; Has as general objective to establish the relationship that exists between an educational program and the level of knowledge about sexuality in adolescents of educational institutions of the district of Moche. The sample consisted of 96 adolescents, who were given two instruments to collect data: The questionnaire to determine the level of knowledge about sexuality and the educational program "Recognizing my adolescence. The data were processed with SPSS Software version 22 In order to establish the relationship between the study variables, the statistical test " $\mathrm{t}$ " of student was applied considering a level of significance of 0.05 , reaching the following conclusions regarding the level of knowledge about sexuality in adolescents before the application of the educational program 78.1\% achieved an average level of knowledge, $17.7 \%$ had a low level and $4.2 \%$ had a high level: after the implementation of the educational program 59.4\% reached an average level of knowledge, 38.5\% a high level and $2.1 \%$ a low level. Regarding the study variables, it was shown that there is a relationship between them.

KEY WORDS: Adolescent; knowledge; sexuality (MeSH).

\section{INTRODUCCIÓN}

La adolescencia es el periodo de la vida en el que la persona adquiere la capacidad reproductiva, transita los patrones psicológicos de la niñez a la edad adulta y consolida la independencia socio económica. Es considerada como una etapa de la vida libre de enfermedades, aunque si se aborda desde la óptica de la salud sexual reproductiva, es importante dotarlo de información suficiente para que comprenda que el ejercicio de la sexualidad durante estos años es riesgoso. Debe manejar sus impulsos adecuadamente y no dejarse llevar por las presiones sociales. El tener información sobre la importancia de planificar el momento de ser padres, es un derecho de los adolescentes, solamente así actuarán de manera responsable, evitarán riesgos y se convertirán en personas sanas, maduras, responsables y confiables ${ }^{1}$. 
La adolescencia sigue siendo ese período de transición y aprendizaje en el que a menudo es difícil acceder a una demanda de información y educación sexual en el que se encuentra el individuo con serios obstáculos para acceder a los anticonceptivos y medidas de prevención de enfermedades de transmisión sexual, lo cual puede acarrear una peor entrada en el mundo de las relaciones sexuales trayendo consecuencias conocidas y alarmantes como el número creciente de embarazos no deseados en menores de edad, matrimonios apresurados, abandono del proceso educativo, riesgos de aborto, alto riesgo de desempleo; sumado a esto el riesgo de contagio de enfermedades de transmisión sexual como el SIDA y la Hepatitis B entre otras que se reportan en cifras considerables entre los adolescentes y adultos jóvenes ${ }^{1}$.

El contexto en que se inicia la actividad sexual en adolescentes tiene importantes significados para las personas e implicancias en salud pública. Los y las adolescentes inician actividad sexual a edades más tempranas que en generaciones anteriores. La evidencia científica muestra que el inicio de la actividad sexual a más temprana edad puede tener consecuencias psicológicas, sociales y económicas negativas. El inicio sexual temprano está asociado también a un mayor número de parejas sexuales a lo largo de la vida. Estas tendencias tienen una influencia enorme en la ocurrencia de comportamientos sexuales de riesgo y en la epidemiología del embarazo adolescente, como también en las infecciones de transmisión sexual y VIH-SIDA².

Una de las consecuencias de no tener una sexualidad segura es adquirir Infecciones de Transmisión Sexual (ITS), el VIH/SIDA, que se contrae cada vez con más frecuencia durante la adolescencia, así como el embarazo no deseado, entre otros; son problemas que limitan la expectativa personal, y afectan su futuro en cuanto a calidad y proyectos de vida ${ }^{3}$.

A nivel de la Región La Libertad (Perú) en el 2013 el 43,24 por ciento de adolescentes de 15-19 años han quedado alguna vez embarazada, el 18,4\% son madres por primera vez, esto ha ido aumentando ya que el año pasado el porcentaje era menor con un 15\%, y lo más resaltante es que existe un $14 \%$ que no han escuchado mensajes sobre salud sexual y reproductiva; además que solo el $4 \%$ de adolescentes fue visitada por un trabajador de salud para hablar sobre planificación familiar y el 84,67\% de adolescentes no usan métodos anticonceptivos y no hablaron sobre planificación familiar con un personal de salud ni en establecimiento de salud ${ }^{4}$. 
Para contrarrestar la problemática antes mencionada se debe mejorar el conocimiento e información de los adolescentes, siendo el conocimiento sinónimo de entendimiento, constituyendo la acción y el efecto de conocer; que comprende el conjunto de saberes sobre determinado tema. Ambos constituyen también el conjunto de datos sobre hechos, verdades o información almacenada a través de la experiencia del aprendizaje (a posteriori) o a través de la introspección (a priori) $)^{5}$.

Todo esto evidencia que los adolescentes requieren de programas especiales que atiendan sus necesidades de salud y muy en particular, aquellos relativos a su conducta sexual y reproductiva. Ahora bien, para lograr buenos resultados en éstos se deben conocer, desde la perspectiva del adolescente, las percepciones sobre su vida sexual y reproductiva, así como educarlos para que asuman comportamientos responsables, sustentados en fuertes vínculos con la pareja, con su familia y la comunidad ${ }^{6}$. Por ese motivo es de interés identificar la efectividad del programa educativo "reconociendo mi adolescencia" en el nivel de conocimiento sobre sexualidad en adolescentes de cuatro instituciones educativas del distrito de Moche, 2015.

\section{Hipótesis}

El programa educativo "reconociendo mi adolescencia" será efectivo si el nivel de conocimiento sobre sexualidad en adolescentes se incrementa en más de un $20 \%$.

\section{METODOLOGÍA}

El estudio es de tipo cuantitativo, pre-experimental, correlacional, de corte transversal. La población estuvo conformada por 252 adolescentes del segundo año de educación secundaria sección "B" de las instituciones educativas Ramiro Nique, Emilio Lefebre, Eulogio Garrido y Nuestra Señora de Guadalupe, todas ubicadas en el distrito de Moche, provincia de Trujillo, Perú; de los cuales se obtuvo una muestra probabilística de 96 adolescentes. Para la recolección de datos se aplicó la técnica de la entrevista, utilizando dos instrumentos: Cuestionario para determinar el nivel de conocimiento sobre sexualidad y el programa educativo "reconociendo mi adolescencia. Instrumentos garantizados en su validez y confiabilidad mediante las pruebas estadísticas de correlación de Pearson y Alpha de Cronbach respectivamente? 


\section{RESULTADOS}

Tabla 1

NIVEL DE CONOCIMIENTO SOBRE SEXUALIDAD ANTES DE LA APLICACIÓN DEL PROGRAMA EDUCATIVO EN ADOLESCENTES. MOCHE, 2015

\begin{tabular}{|c|c|c|c|c|c|c|c|c|c|c|}
\hline \multirow{3}{*}{$\begin{array}{c}\text { Nivel de } \\
\text { Conocimientos }\end{array}$} & \multicolumn{8}{|c|}{ Colegios } & \multirow{2}{*}{\multicolumn{2}{|c|}{ Total }} \\
\hline & \multicolumn{2}{|c|}{$\begin{array}{l}\text { Emilio } \\
\text { Lefebre }\end{array}$} & \multicolumn{2}{|c|}{$\begin{array}{l}\text { Ramiro } \\
\text { Nique } \\
\text { Espíritu }\end{array}$} & \multicolumn{2}{|c|}{$\begin{array}{l}\text { Nuestra } \\
\text { Señora de } \\
\text { Guadalupe }\end{array}$} & \multicolumn{2}{|c|}{$\begin{array}{l}\text { Eulogio } \\
\text { Garrido }\end{array}$} & & \\
\hline & $n^{\circ}$ & $\%$ & $n^{\circ}$ & $\%$ & $n^{\circ}$ & $\%$ & $n^{\circ}$ & $\%$ & $n^{\circ}$ & $\%$ \\
\hline Bajo & 4 & 12,5 & 5 & 22,7 & 4 & 16,0 & 4 & 23,5 & 17 & 17,7 \\
\hline Medio & 28 & 87,5 & 15 & 68,2 & 19 & 76,0 & 13 & 76,5 & 75 & 78,1 \\
\hline Alto & 0 & 0,0 & 2 & 9,1 & 2 & 8,0 & 0 & 0,0 & 4 & 4,2 \\
\hline Total & 32 & 100,0 & 22 & 100,0 & 25 & 100,0 & 17 & 100,0 & 96 & 100,0 \\
\hline
\end{tabular}

Fuente: Datos obtenidos del cuestionario para determinar el nivel de conocimiento sobre sexualidad.

Tabla 2

NIVEL DE CONOCIMIENTO SOBRE SEXUALIDAD DESPUES DE LA APLICACIÓN DEL PROGRAMA EDUCATIVO EN ADOLESCENTES. MOCHE, 2015

\begin{tabular}{|c|c|c|c|c|c|c|c|c|c|c|}
\hline \multirow{3}{*}{$\begin{array}{c}\text { Nivel de } \\
\text { Conocimientos }\end{array}$} & \multicolumn{8}{|c|}{ Colegios } & \multirow{2}{*}{\multicolumn{2}{|c|}{ Total }} \\
\hline & \multicolumn{2}{|c|}{$\begin{array}{l}\text { Emilio } \\
\text { Lefebre }\end{array}$} & \multicolumn{2}{|c|}{$\begin{array}{l}\text { Ramiro } \\
\text { Nique } \\
\text { Espíritu }\end{array}$} & \multicolumn{2}{|c|}{$\begin{array}{l}\text { Nuestra } \\
\text { Señora de } \\
\text { Guadalupe }\end{array}$} & \multicolumn{2}{|c|}{$\begin{array}{l}\text { Eulogio } \\
\text { Garrido }\end{array}$} & & \\
\hline & $n^{\circ}$ & $\%$ & $n^{\circ}$ & $\%$ & $n^{\circ}$ & $\%$ & $n^{\circ}$ & $\%$ & $n^{\circ}$ & $\%$ \\
\hline Bajo & 0 & 0,0 & 1 & 4,5 & 1 & 4,0 & 0 & 0,0 & 2 & 2,1 \\
\hline Medio & 18 & 56,3 & 15 & 68,2 & 14 & 56,0 & 10 & 58,8 & 57 & 59,4 \\
\hline Alto & 14 & 43,8 & 6 & 27,3 & 10 & 40,0 & 7 & 41,2 & 37 & 38,5 \\
\hline Total & 32 & 100,0 & 22 & 100,0 & 25 & 100,0 & 17 & 100,0 & 96 & 100,0 \\
\hline
\end{tabular}

Fuente: Datos obtenidos del cuestionario para determinar el nivel de conocimiento sobre sexualidad. 
La tabla 1 muestra el nivel de conocimientos sobre sexualidad que tienen los adolescentes de las Instituciones Educativas en estudio antes de la aplicación del programa educativo. Reportando que el 78,1\% alcanzó un nivel medio de conocimientos, el 17,7\% un nivel bajo y solo el 4,2\% un nivel alto.

La tabla 2 muestra el nivel de conocimientos sobre sexualidad que tienen los adolescentes de las Instituciones Educativas en estudio después de la aplicación del programa educativo. Reportando que el 59,4\% alcanzó un nivel medio de conocimientos, el 38,5\% un nivel alto y solo el 2,1\% un nivel bajo.

Tabla 3

EFECTIVIDAD DEL PROGRAMA EDUCATIVO "RECONOCIENDO MI ADOLESCENCIA” EN EL NIVEL DE CONOCIMIENTO SOBRE SEXUALIDAD

EN ADOLESCENTES DE LAS INSTITUCIONES EDUCATIVAS RAMIRO NIQUE, EMILIO LEFEBRE, NUESTRA SEÑORA DE GUADALUPE Y EULOGIO GARRIDO. MOCHE, 2015

\begin{tabular}{ccccc}
\hline Colegios & Variable & $\begin{array}{c}\text { Valor } \\
\text { tstudent }\end{array}$ & $\begin{array}{c}\text { Probabilidad } \\
(p)\end{array}$ & Significancia \\
\hline $\begin{array}{c}\text { Emilio } \\
\text { Lefebre }\end{array}$ & $\begin{array}{c}\text { Conocimiento sobre } \\
\text { sexualidad }\end{array}$ & $-9,724$ & 0,000 & $\begin{array}{c}\text { Altamente } \\
\text { Significativo }\end{array}$ \\
\hline $\begin{array}{c}\text { Ramiro } \\
\text { Nique } \\
\text { Espíritu }\end{array}$ & $\begin{array}{c}\text { Conocimiento sobre } \\
\text { sexualidad }\end{array}$ & $-3,568$ & 0,002 & $\begin{array}{c}\text { Altamente } \\
\text { Significativo }\end{array}$ \\
\hline $\begin{array}{c}\text { Nuestra } \\
\text { Señora de } \\
\text { Guadalupe }\end{array}$ & $\begin{array}{c}\text { Conocimiento sobre } \\
\text { sexualidad }\end{array}$ & $-4,365$ & 0,000 & $\begin{array}{c}\text { Altamente } \\
\text { Significativo }\end{array}$ \\
\hline $\begin{array}{c}\text { Eulogio } \\
\text { Garrido }\end{array}$ & $\begin{array}{c}\text { Conocimiento sobre } \\
\text { sexualidad }\end{array}$ & $-7,156$ & 0,000 & $\begin{array}{c}\text { Altamente } \\
\text { Significativo }\end{array}$ \\
\hline
\end{tabular}

Fuente: Datos obtenidos del cuestionario para determinar el nivel de conocimiento sobre sexualidad.

En la tabla 3 se observa el resultado de la prueba estadística (T Student), el cual es altamente significativo relacionando el puntaje de conocimiento sobre sexualidad antes y después de la aplicación del programa educativo en las y los adolescentes de los cuatro colegios del distrito del Moche, 2015. 


\section{DISCUSIÓN}

La sexualidad es el modo en el que el ser humano tiene que vivirse así mismo, es la forma de verse y de sentirse como hombre o como mujer. Cada uno tiene su propio modo de vivir, asumir, potenciar y cultivar su sexualidad. Los adolescentes deben asumir que son seres sexuales, puesto que se trata de la faceta de una persona, la sexualidad está presente durante toda la vida, desde que nace hasta que muere, aunque se mantiene de forma diferente en cada etapa de su vida ${ }^{8}$.

La sexualidad constituye sin lugar a dudas, un importante desafío para todos los que, desde la perspectiva de la educación integral, persiguen un mejoramiento de la calidad de vida y el perfeccionamiento de los modos de actuación en torno a la vida sexual y reproductiva, en las parejas y en las familias. Se conoce que la sexualidad humana depende menos de los mecanismos fisiológicos y mucho más de los contextos sociales, donde el aprendizaje constituye un aspecto esencial. Por ello el propio desarrollo social ha conducido al inicio precoz de las relaciones sexuales en adolescentes de ambos sexos ${ }^{6}$.

La tabla 1, muestra el nivel de conocimientos sobre sexualidad que tienen los adolescentes de las Instituciones Educativas en estudio antes de la aplicación del programa educativo. El 78,1\% de estas alcanzó un nivel medio de conocimientos, el 17,7\% un nivel bajo y solo el 4,2\% un nivel alto.

Estos resultados son semejantes a los encontrados por Carrasco y Castro en el 2008, en la investigación sobre conocimiento de sexualidad en adolescentes de 4to y 5 to de secundaria en una Institución educativa del distrito de La Esperanza, donde encontraron en el pre test que el $60 \%$ de los adolescentes obtuvieron un nivel de conocimientos sobre sexualidad medio ${ }^{9}$.

Asimismo, Murga en el 2011, en su investigación sobre Conocimientos y Prácticas sobre Sexualidad e Impulsos Sexuales en los Adolescentes en la I.E.6041 Alfonso Ugarte de San Juan de Miraflores, en el año 2011"10. Encontró un porcentaje considerable de los adolescentes conoce sobre sexualidad, cambios físicos/corporales del adolescente, rol sexual y masturbación, un porcentaje considerable de los adolescentes tiene prácticas sexuales seguras ya que la mayoría tienen relaciones con una sola pareja y la persona con quien inician su relación sexual es segura mientras que un considerable porcentaje significativo que tiene prácticas sexuales riesgosas referidas al inicio de las relaciones sexuales. 
Por el contrario, Sánchez y Valdivieso en su investigación Mejorando mis saberes sobre salud sexual y reproductiva realizado en la Institución Educativa Liceo Trujillo, encontraron en el pre test que el 68,7\% de los adolescentes tenían un nivel de conocimiento sobre sexualidad alto ${ }^{11}$.

La tabla 2 muestra el nivel de conocimientos sobre sexualidad que tienen los adolescentes de las Instituciones Educativas del distrito de Moche, después de la aplicación del programa educativo, el 59,4\% de estas alcanzó un nivel medio de conocimientos, el 38,5\% un nivel alto y solo el 2,1\% un nivel bajo. En un estudio realizado por Condori en el 2008, titulado, "Efectividad de un programa educativo en el nivel de conocimientos sobre ITS y VIH/SIDA en estudiantes de la Institución Educativa Héroes de la Breña, Lima. Encontró que después de ser aplicado el programa educativo, el $95 \%$ tuvieron conocimiento alto, resultados que difieren a los de la presente investigación ${ }^{12}$.

De la Cruz y Gasco en el 2014 investigaron sobre la Efectividad del programa educativo "conversando sobre sexualidad" en el nivel de conocimiento de los adolescentes, Trujillo, encontraron que después de su aplicación del programa educativo, el 92,5\% de los adolescentes de la Institución Educativa Emblemática José Faustino Sánchez Carrión, presentaron un nivel de conocimiento sobre sexualidad alto, resultados que difieren de los de la presente investigación ${ }^{13}$.

En la tabla 3 se observa el resultado de la prueba estadística aplicada (T Student), la cual es altamente significativa relacionando el puntaje de conocimiento sobre sexualidad antes y después de la aplicación del programa educativo en las y los adolescentes del distrito de Moche, 2015. Los resultados de la presente investigación son similares a los reportados por Sandoval, Restrepo, Gallo y Molina, en su investigación sobre, evaluación del impacto del programa de Salud Sexual y Reproductiva en adolescentes de Medellín. Ellos demostraron la efectividad en el incremento de conocimientos especialmente del grupo femenino, un incremento general en el uso de métodos anticonceptivos, 5 de cada diez refirieron el uso de métodos anticonceptivos en la primera relación sexual y para evitar el embarazo ${ }^{14}$.

Cabray en el 2010, luego de sus investigaciones en Adolescencias y Juventudes, recomienda que las intervenciones se realicen en las familias o en los centros educativos, por su bajo costo, por su valor saludable y básicamente por el desarrollo integral del ser humano. Aclara, que educar no es imprimir lo que uno sabe en el otro, sino muy por el contrario, es conducir hacia afuera lo que existe adentro de él. No se trata de asumir no rechazar valores sociales establecidos, ni tampoco 
de retocar ni perfeccionar aquello que está mal, el gran desafío es la posibilidad de construir un diálogo continuo y fecundo ${ }^{15}$.

Uno de los conceptos importantes de la sexualidad es la Salud sexual y reproductiva, que viene a ser el estado general de bienestar físico, mental y social de las personas y no la ausencia de enfermedades o dolencias, en la esfera sexual y reproductiva, así como sus funciones y procesos. Las personas son capaces de disfrutar de una vida sexual satisfactoria y sin riesgos en la que no es obligatoria la procreación, pues la sexualidad en sí misma es una forma de comunicación, convivencia y/o amor entre las personas ${ }^{5}$.

\section{CONCLUSIONES}

1. Respecto al nivel de conocimiento sobre sexualidad en adolescentes antes de la aplicación del programa educativo el 78,1\% alcanzó un nivel medio de conocimientos, el $17,7 \%$ un nivel bajo y el $4,2 \%$ un nivel alto.

2. Después de la aplicación del programa educativo el 59,4\% alcanzó un nivel medio de conocimientos, el $38,5 \%$ un nivel alto y el $2,1 \%$ un nivel bajo.

3. En relación a las variables de estudio se demostró que existe relación estadística altamente significativa entre ellas.

\section{REFERENCIAS BIBLIOGRÁFICAS}

1. Organización Mundial de la Salud. El embarazo en la adolescencia [Internet]. 2018 [cited 2018 May 9]. Available from:

http://www.who.int/es/news-room/fact-sheets/detail/adolescent-pregnancy

2. González A E, Molina G T, Montero A, Martínez N V, Leyton M C. Comportamientos sexuales y diferencias de género en adolescentes usuarios de un sistema público de salud universitario. Rev Med Chil [Internet]. 2007 Oct [cited 2018 May 9];135(10):1261-9. Available from: http://www.scielo.cl/scielo.php?script=sci_arttext\&pid=S0034 . $98872007001000005 \& \operatorname{lng}=\mathrm{en} \& n r m=$ iso\&tlng $=$ en

3. Monroy de Velasco A. Salud y sexualidad en la adolescencia y juventud: guía práctica para padres y educadores [Internet]. 1ra edició. Mexico: Editorial Pax México; 2002 [cited 2018 May 9].256 p. Available from: https://archive.org/details/isbn_9789688605073

4. Instituto Nacional de Estadística e Informática del Perú. Adolescente [Internet]. Presidencia del Consejo de Ministros del Perú. 2017 [cited 2018 May 9]. Available from: https://www.inei.gob.pe/buscador/?tbusqueda = adolescente 
5. Dirección General de Promoción de la Salud. Plan de implementación de eje temático de: Salud sexual y reproductiva en el programa de promoción de la salud en las Instituciones Educativas. Lima: Ministerio de Salud del Perú; 2005.

6. Rodríguez A, Álvarez L. Percepciones y comportamientos de riesgos en la vida sexual y reproductiva de los adolescentes. Rev Cuba Salud Pública [Internet]. 1988 [cited 2018 May 9];32(1):1-9. Available from: http://www.redalyc.org/articulo.oa?id=21432108

7. Hernández S. Metodología de la Investigación. México; 2006.

8. Barrero L. Sexualidad Humana. Mexico; 2012.

9. Carrasco P, Castro M. Nivel de conocimientos sobre sexualidad en adolescentes de los centros educativos del Distrito de la Esperanza. Universidad Nacional de Trujillo; 2008.

10. Murga S. Conocimientos y prácticas sobre sexualidad e impulsos sexuales en los adolescentes en la I.E. 6041, Alfonso Ugarte. Universidad Nacional Mayor de San Marcos; 2011.

11. Sánchez C, Valdivieso A. Mejorando mis saberes sobre salud sexual y reproductiva. Universidad Nacional de Trujillo; 2011.

12. Condori H. Efectividad de un programa educativo en el nivel de conocimientos sobre ITS y VIH/SIDA en estudiantes de la Institución Educativa Héroes de la Breña en el año 2008 [Internet]. Universidad Nacional Mayor de San Marcos; 2009 [cited 2018 May 9].

Available from: http://cybertesis.unmsm.edu.pe/bitstream/handle/cybertesis/487/ Condori_ph.pdf?sequence $=1$

13. De la Cruz D, Gasco R. Efectividad del programa educativo "Conversando sobre Sexualidad" en el nivel de conocimiento de los adolescentes, Trujillo. Universidad Nacional de Trujillo; 2014.

14. García G, Sandoval J, Restrepo A, Gallo N, Molina N. Evaluación del impacto del programa de Salud Sexual y Reproductiva en Adolescentes de Medellín, 2008 [Internet]. Medellin: Alcaldía de Medellín; 2008 [cited 2018 May 9]. Available from: https://www.medellin.gov.co/irj/go/km/docs/wpccontent/Sites/Subportal del Ciudadano/Salud/Secciones/Publicaciones/Documentos/2012/Investigaciones/Salud sexual y reproductiva 2008.pdf

15. Crabay MI. Adolescencias y juventudes: subjetividades y riesgos [Internet]. 1ra ed. Córdova: Editorial Brujas; 2016 [cited 2018 May 9]. 350 p. Available from:

http://www.editorialbrujas.com.ar/detalle.php?idlibro $=374$ 